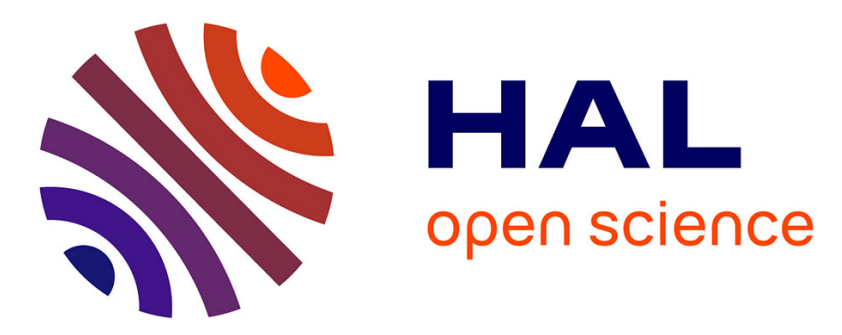

\title{
Percolation of holes between overlapping spheres : Monte Carlo calculation of the critical volume fraction
}

\author{
J. Kertész
}

\section{To cite this version:}

J. Kertész. Percolation of holes between overlapping spheres: Monte Carlo calculation of the critical volume fraction. Journal de Physique Lettres, 1981, 42 (17), pp.393-395. 10.1051/jphyslet:019810042017039300 . jpa-00231955

\section{HAL Id: jpa-00231955 https://hal.science/jpa-00231955}

Submitted on 1 Jan 1981

HAL is a multi-disciplinary open access archive for the deposit and dissemination of scientific research documents, whether they are published or not. The documents may come from teaching and research institutions in France or abroad, or from public or private research centers.
L'archive ouverte pluridisciplinaire HAL, est destinée au dépôt et à la diffusion de documents scientifiques de niveau recherche, publiés ou non, émanant des établissements d'enseignement et de recherche français ou étrangers, des laboratoires publics ou privés. 


\title{
LE JOURNAL DE PHYSIQUE-LETTRES
}

\section{Percolation of holes between overlapping spheres : Monte Carlo calculation of the critical volume fraction}

\author{
J. Kertész $(*)$ \\ Institute for Theoretical Physics, University of Cologne, Zülpicher Strasse 77, D-5000 Köln 41, West Germany
}

(Reçu le 25 mai 1981, accepté le 16 juillet 1981)

\begin{abstract}
Résumé. - On utilise une méthode de simulation de type Monte Carlo pour calculer le seuil de percolation des trous entre sphères en recouvrement. Le volume critique, $0,966 \pm 0,007$ correspondant à la densité sans dimension $0,81 \pm 0,05$, est le point où apparaît la localisation dans le modèle de Lorentz.
\end{abstract}

Abstract. - A Monte Carlo method is used to calculate the threshold of percolation of holes between overlapping spheres. At the critical volume fraction, $0.966 \pm 0.007$, corresponding to a dimensionless density of $0.81 \pm 0.05$ localization in the Lorentz model appears.

1. Introduction. - Originally percolation theory was constructed to describe, for example, how a fluid spreads through a porous medium $[1,2]$. These phenomena do not necessarily take place on a regular lattice; however, most of the calculations were carried out on lattices because the methods used are much more effective [3, 4]. One can alternatively consider percolation on a continuum, where the volume fraction $p(\leqslant 1)$ of " black " material is somehow controlled [5]. A cluster is then defined as that part of the "black " material which can be reached from a " black " point without touching any « white " material. The simplest way to construct such a system is to consider identical noninteracting "black " discs (in two dimensions) or spheres (in three dimensions) at a given density. Two discs (spheres) belong to the same cluster if there is a path through overlapping discs (spheres) from one to the other. Percolation appears if the density is high enough to have an infinite " black » cluster [6]. It has been shown recently, that this model probably belongs to the same universality class as percolation on a lattice [7-9], i.e. the critical exponents are the same. This justifies the use of the results on universal quantities for continuum percolation. However, the critical point is nonuniversal and it should be determined separately for each model of interest.

(*) Permanent address : Institute for Technical Physics of the Hungarian Academy of Sciences, Budapest, Ujpest 1, Pf. 76, H-1325, Hungary.
For any "black» percolation problem one can define a complementary one where the percolation of "white " material is investigated. Obviously, if the geometry of "black " and "white " clusters is the same, the volume fraction, corresponding to the critical point of "white " material $\left(p_{\mathrm{C}}^{\mathbf{w}}\right)$ is simply related to the « black» threshold $\left(p_{\mathrm{C}}^{\mathrm{B}}\right)[5]$ :

$$
p_{\mathrm{C}}^{\mathbf{W}}=1-p_{\mathrm{C}}^{\mathrm{B}} \text {. }
$$

This is the case for example for lattice (site) percolation. In the problem of discs (spheres) the geometry of " white " holes between the "black " discs (spheres) is different from that of the "black " material and so equation (1) does not hold. In two dimensions the "black" and "white" infinite clusters cannot exist at the same time [5]. On the other hand, if the «black " infinite cluster is cut by the " white " material the " white " infinite cluster immediately appears and vice versa, i.e.

$$
p_{\mathrm{C}}^{\mathbf{W}}=p_{\mathrm{C}}^{\mathbf{B}} .
$$

(For more detailed discussion of this problem see reference [5].) These arguments do not hold for the three dimensional case, where the "black" and " white " infinite clusters can exist at the same time.

In this letter the percolation of "white" holes between overlapping identical "black" spheres is investigated. This model should be relevant to the original percolation problem (how a fluid spreads through a porous medium) [2], or to the problem of gas permeability in a compressed powder, an important 
phenomenon in the sintering process [10]. Another related problem is the localization in the Lorentz model for the classical motion of a particle in free space between randomly distributed hard-sphere scatterers. At a special density localization appears if the spheres are allowed to overlap [11]. The volume fraction which corresponds to this threshold density $\left({ }^{1}\right)$ is the critical point $p_{\mathrm{C}}^{\mathbf{W}}$. For the two dimensional Lorentz model this threshold was found by computer simulations and equation (2) was confirmed [12]. For the three dimensional case only theoretical predictions exist [11] because the computer experiments have not been carried out at high enough densities [13]. The present work is the determination of the critical point by Monte Carlo calculations in three dimensions.

2. Method. - First a given number $(N)$ of « black » spheres are put randomly into a unit cube with periodic boundary conditions. The coordinates of the centres of the spheres are determined using random numbers (CC «RANF») equally distributed between 0 and 1 , so there is no correlation among these centres. Then the radius of the spheres is varied. The volume fraction $p_{\mathrm{C}}^{\mathrm{w}}(N)$ where the path of "white " material from the top to the bottom is first blocked by a sphere is the threshold point of the given sample. The quantity $p_{\mathrm{C}}^{\mathrm{w}}(N)$ depends both on the configuration of the $N$ particles and on $N$ itself. The mean $\left\langle p_{\mathrm{C}}^{\mathrm{w}}(N)\right\rangle$ can be identified with the threshold belonging to size $N$. In order to obtain $p_{\mathrm{C}}^{\mathrm{W}}(N)$, a simple cubic lattice is superimposed on the system with lattice constant $a=1 /(L-1)$, i.e. the lattice consists of $L^{3}$ sites. Each site can have two states : occupied if it is located in " white " material and empty if it is located in " black ». Filling up the lattice in this manner we have a (correlated) lattice percolation problem for which well-developed numerical methods [14] can be used to find the radius where percolation vanishes. For a given radius the computer checks whether there is a spanning cluster. The computer program is based on a block diagram of reference [14]; detailed description of a nonoptimized version will be given in reference [15]. The radius, where percolation first appears, is confined using iterations [16]. By making the lattice finer, in the limit $a \rightarrow 0$ one gets $p_{\mathrm{C}}^{\mathrm{W}}(N)$. Single bits were used to store information about the states of the lattice sites and so it was possible to use lattices up to $L=95$. A single run with $L=95$, $N=2000$ took about $32 \mathrm{~s}$ on the CDC Cyber $76 \mathrm{M}$. Usually 7 iterations [15] were needed to determine the threshold point for a given configuration, and 36 configurations were generated for $N=100,200,300,400$ and 12 for $N=2000$. An extrapolation according to the $a \rightarrow 0$ limit was carried out, using $L=30,40$,

( $\left.{ }^{1}\right)$ The volume fraction of spheres (" black " material) is simply related to the dimensionless density : $p=1-\exp \left(-4 \pi n^{*} / 3\right)$, $n^{*}=r^{3} n, r$ is the radius of spheres, $n$ their density.
$50,60,75,85,95$ for $N=100$ and $L=50,75,85,95$ for $N=200,300,400,2000$. After calculating $p_{\mathrm{C}}^{\mathrm{W}}(N)$ for various values of $N$, another extrapolation is needed to obtain the threshold value of the infinite system :

$$
p_{\mathbf{C}}^{\mathbf{W}}=\lim _{N \rightarrow \infty}\left\langle p_{\mathbf{C}}^{\mathbf{W}}(N)\right\rangle
$$

3. Results and discussion. - It is important to point out that the $a \rightarrow 0$ extrapolation is for a given configuration, which means for various lattice constants $N$ is fixed and the same random numbers are used to place the spheres. In order to see how the $a \rightarrow 0$ extrapolation can be carried out, we generated 36 configurations with $N=100$ and 7 different values of $a$. Figure 1 shows that data can be extrapolated by a straight line if plotted against $1 / L$. Thus the same type of extrapolation was applied for the remaining system sizes, but only four values of $L$ were chosen. The resulting $\left\langle p_{\mathrm{C}}^{\mathbf{w}}(N)\right\rangle$ values are plotted in figure 2 , with the estimated error bars, against $1 / N$. Usually, according to finite size scaling [15], the limiting process is taken as a straight line extrapolation of $\left\langle p_{\mathrm{C}}^{\mathrm{W}}(N)\right\rangle$ versus $N^{-1 / 3 v}$ (with the correlation length exponent $v$ ), but we do not

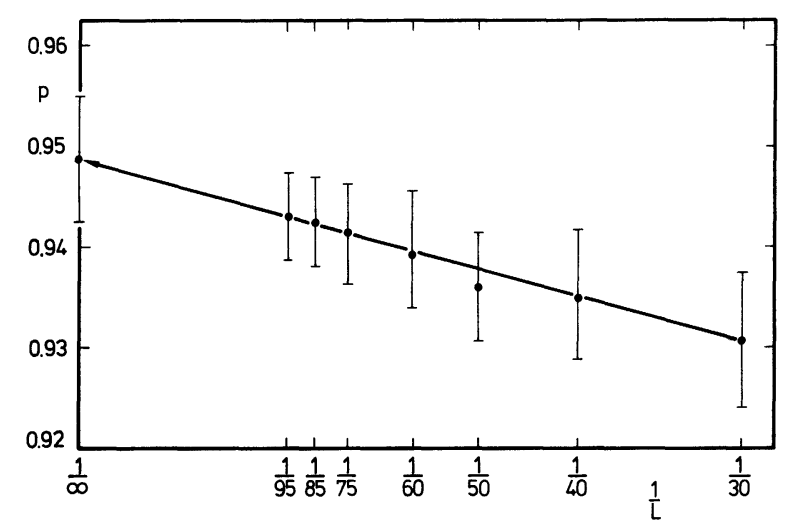

Fig. 1. - Extrapolation to the finite size continuum limit with $N=100$ spheres. The auxiliary lattice has the lattice constant $a=1 /(L-1)$.

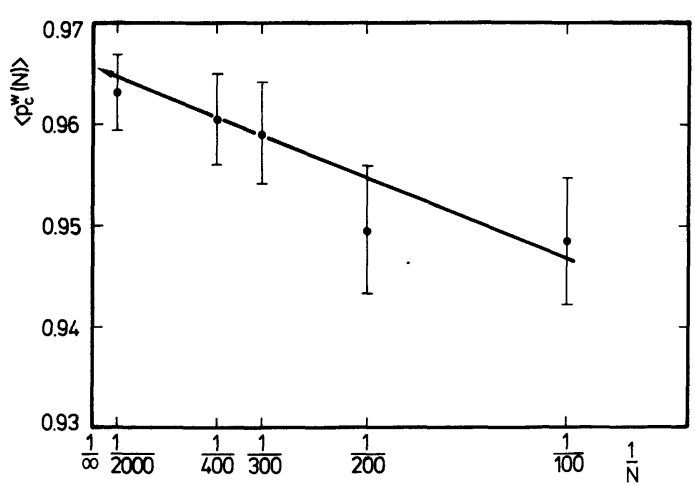

Fig. 2. - Extrapolation to the infinite size limit $(N \rightarrow \infty)$. The estimated value of $p_{\mathrm{C}}^{\mathrm{W}}$ is $0.966 \pm 0.007$. 
think that our data are accurate enough to be sensitive to the way of extrapolation. As a result we obtain

$$
p_{\mathrm{C}}^{\mathbf{W}}=0.966 \pm 0.007
$$

It should be mentioned that the overall trend in $p_{\mathrm{C}}^{\mathrm{W}}(N)$ becomes clear only after taking the $a \rightarrow 0$ limit.

To test the method we calculated also the percolation threshold for overlapping spheres $\left(p_{\mathrm{C}}^{\mathbf{B}}\right)$ in the same way. We obtained $p_{C}^{\mathrm{B}}=0.280 \pm 0.007$ which should be compared with the value between 0.283 and 0.295 (for references see [7]). There is one early calculation [17] which led to extremely low values of $p_{\mathrm{C}}^{\mathrm{B}}(0.256)$. In this work a method somewhat similar to ours was used. However from our result one can see that by enlarging the sample size and the number of lattice points, the extrapolation procedure can be improved and the obtained threshold is shifted upwards.

The critical volume fraction (4) corresponds to a dimensionless density $n^{*}=n r^{3}=0.81 \pm 0.05$. At this point localization should appear in the Lorentz model. Unfortunately, there is no computer simulation at densities high enough. If Bruin's data on the diffusion coefficient [13] are extrapolated linearly versus $n^{*}$ one gets the localization density at about $n^{*} \sim 0.72$. We think that this linear extrapolation corresponds to a mean field approximation and deviation from it may appear at higher densities, as indicated by one data point quoted in [11]. The theory of Götze et al. [11] based on the memory function technique leads to an $n^{*}=0.716$, which is in very good agreement with the linear extrapolation of Bruin's data and is thus somewhat outside of our error bars; however, the theory appears to work much better in three dimensions than in two, where $n^{*}=0.37$ (computer simulation, [12]) and $n^{*}=0.637$ (theory, [11]). The memory function technique seems to be a kind of mean field theory : it is better in higher dimensions but near to the critical point there are still aberrations.

4. Conclusion. - We investigated the percolation of holes between overlapping spheres by Monte Carlo technique. Two kinds of extrapolations were used : one for the continuum limit of the finite size samples and the other for obtaining the infinite size limit. The critical point is at a volume fraction $p_{\mathrm{C}}^{\mathbf{W}}=0.966 \pm 0.007$ of spheres, i.e. above this threshold the percolation between the spheres is not possible. As far as we know this has been the first such calculation for a continuum percolation problem without underlying graph structure.

Acknowledgments. - We are grateful to D. Stauffer for his valuable advice, as well as to him and B. K. Chakrabarti for their critical reading of the manuscript. In addition we wish to thank W. Götze and E. Leutheusser for interesting discussions on the Lorentz model. The financial support of the German Academic Exchange Service (DAAD) is gratefully acknowledged. We thank J. Roussenq for the French abstract.

\section{References}

[1] Broadbent, S. and Hammersley, J., Proc. Cambridge Philos. Soc. 53 (1957) 629.

[2] De Gennes, P. G. and Guyon, E., J. Mécanique 17 (1978) 1.

[3] Stauffer, D., Phys. Rep. 54 (1979) 1.

[4] Essam, J. W., Rep. Prog. Phys. 43 (1980) 833.

[5] Zallen, R. and Scher, H., Phys. Rev. B 4 (1971) 4471.

[6] Roberts, F. D. K., Biometrika 54 (1967) 625.

[7] HaAn, S. W. and Zwanzig, R., J. Phys. A 10 (1977) 1547.

[8] Vicsex, T. and Kertész, J., J. Phys. A 14 (1981) L 31.

[9] Gawlinsky, E. T. and Stanley, H. E., preprint.

[10] Lacour, C., Phys. Status Solidi (b) 96 (1979) 785.

[11] Götze, W., Leuthheussen, E. and YIP, S., Phys. Rev. A 23 (1981) 2634, and ibid, in print.
[12] Alder, B. J. and Alley, W. E., J. Stat. Phys. 19 (1978) 341.

[13] Bruin, C., Physica 72 (1974) 261.

[14] Hoshen, J. and Kopelman, R., Phys. Rev. B 14 (1976) 3438.

[15] StAuffer, D., in International Conference on Disordered Systems and Localization, Proceedings to be published in Springer Lecture Notes on Physics Series, eds. C. Castellani, C. Di Castro and L. Peliti, (Springer Verlag, Heidelberg) 1981.

[16] Levinshtein, M. E., Shklovskil, B. I., ShuR, M. S. and EFros, A. L., Zh. Exp. Theor. Fis. 69 (1975) 386.

[17] Hоlсомв, D. F. and ReHR Jr., J. J., Phys. Rev. 183 (1969) 773. 